\title{
Riqueza e estratificação vertical de epífitas vasculares na Estação Ecológica de Jataí - área de Cerrado no Sudeste do Brasil
}

\author{
Fernando Antonio Bataghin ${ }^{1,3}$, Adelcio Muller ${ }^{1}$, José Salatiel Rodrigues Pires ${ }^{1}$, Fábio de Barros ${ }^{2}$, \\ Angela Terumi Fushita ${ }^{1}$ e Eliziane Carla Scariot ${ }^{1}$
}

Recebido: 4.10.2012; aceito: 6.12 .2012

\begin{abstract}
Richness and vertical stratification of vascular epiphytes in Jataí Ecological Station - a Southeast Brazilian Cerrado area). The richness and vertical stratification of vascular epiphytes was studied in Jataí Ecological Station (EEJ), São Paulo State, Brazil. A total of 1,574 epiphytes were recorded, corresponding to 29 species, 20 genera, and seven families. Angiosperms were represented by 23 species and Pteridophytes by six species. The characteristic form, holoepiphytes, was dominant in the area (93.1\%). Epiphytes specialists were most common in the area, contributing with about 55\% of species (17\% trunk epiphytes and 38\% canopy epiphytes); generalist species contributed $42 \%$, and hemiepiphytes, with $3 \%$. The intermediate zones had the highest number of species and more number of records. There was a significant difference among species that comprise the lower zones and the upper zones in the host trees. The richness and distribution of epiphytes are determined by some climatic factors, but the moisture and light intensity are the most important regulatory factors in the cerrado area researched.
\end{abstract}

Key words: Ecological types, growth form, hotspots, phytosociology of epiphytes

RESUMO - (Riqueza e estratificação vertical de epífitas vasculares na Estação Ecológica de Jataí - área de Cerrado no Sudeste do Brasil). A riqueza e a estratificação vertical das epífitas vasculares foram estudadas na Estação Ecológica de Jataí (EEJ), SP, Brasil. No total, 1.574 epífitas pertencentes a 29 espécies, 20 gêneros e sete famílias foram encontradas. As Angiospermas foram representadas por 23 espécies e as Samambaias, por seis espécies. Os holoepífitos característicos formam dominantes na área (93,1\%). Epífitas especialistas foram predominantes na área, cerca de 55\% das espécies (17\% de epífitas de fuste e $38 \%$ de epífitas de copa); as espécies generalistas contribuíram com cerca de $42 \%$ e as hemiepífitas com 3\%. As zonas intermediárias apresentaram maior número de espécies e maior número de registros. Houve diferença significativa entre as espécies que compõem as zonas inferiores e as zonas superiores nos forófitos. A riqueza e a distribuição de epífitas são determinadas pelos fatores microclimáticos, sendo a umidade e a intensidade luminosa os fatores reguladores mais importantes na área de Cerrado estudada.

Palavras-chave: Fitossociologia de epífitas, forma de crescimento, hotspots, tipos ecológicos

\section{Introdução}

Epífitas vasculares são importantes componentes da biodiversidade em florestas tropicais, e têm atraído a atenção de pesquisadores desde o século XIX (Schimper 1888, Benzing 1990, Barthlott et al. 2001, Krömer et al. 2007). As epífitas são responsáveis por $10 \%$ das plantas vasculares, quase 25.000 espécies (Kress 1986) e, em algumas florestas, pela metade do total do número de indivíduos (Wolf 1994, Nieder et al. 2001).
A importância das epífitas vasculares deve-se ao número de espécies que elas perfazem, à biomassa que acumulam (Gentry \& Dodson 1987, Benzing 1990), à sua participação na ciclagem de nutrientes nos ecossistemas (Edwards \& Grubb 1977, Nadkarni 1992), ao fornecimento de hábitat e alimento para uma variada gama de insetos e pássaros (Benzing 1990, Lugo \& Scatena 1992), além da sua utilidade para o homem, como medicamentos, na agricultura e horticultura (Nadkarni 1992).

1. Universidade Federal de São Carlos, Centro de Ciências Biológicas e da Saúde, Departamento de Hidrobiologia, Via Washington Luiz, km 235, 13565-905 São Carlos, SP, Brasil

2. Instituto de Botânica, Núcleo de Pesquisa Orquidário do Estado, Caixa Postal 68041, 04045-972 São Paulo, SP, Brasil

3. Autor para correspondência: bataghin@ufscar.br 
A distribuição espacial das epífitas vasculares depende das espécies, idade e diâmetro de seus forófitos (Zotz et al. 2001, Arévalo \& Betancur 2006, Flores-Palacios \& García-Franco 2006, Zots \& Schultz 2008), além das condições micro-climáticas (Johansson 1974, Freiberg 1996). Fatores como temperatura, umidade, incidência e composição do espectro de luz, e polarização dos raios são importantes para a flora epifítica e variam de forma diferenciada dentro da floresta (Benzing 1995).

O estabelecimento das epífitas vasculares no gradiente vertical pode estar relacionado com adaptações ecofisiológicas às variações de luminosidade e umidade (Johansson 1974, Arévalo \& Betancur 2006, Krömer et al. 2007), ou às variações nesses fatores desde o dossel até o solo (Parker 1995). A troca de espaços, em termos de insolação, por condições de maior estresse para aquisição de água e nutrientes foi a característica marcante para o aparecimento das epífitas (Benzing 1990). O fato de o estresse hídrico ser limitante para as epífitas, faz com que elas sejam observadas principalmente em florestas úmidas; a aridez exclui a maioria das espécies de epífitas vasculares (Kersten 2006). A exposição das epífitas a elevados índices de insolação, grandes flutuações de temperatura e umidade, além de variação na quantidade de água disponível, torna a sobrevivência dessas dependente de adaptações, tanto nos aspectos morfológicos como fisiológicos (Kira \& Yoda 1989).

Embora haja um crescente interesse no estudo do dossel (Nadkarni et al. 2011), especialmente em relação às epífitas vasculares e sua contribuição para a biodiversidade e conservação (Köster et al. 2009), pouco tem sido publicado sobre as epífitas vasculares em áreas de Cerrado no Brasil, especialmente sobre a dinâmica de distribuição vertical da sinúsia epifítica.

A alta biodiversidade do Cerrado deve-se à sua vasta extensão e fez com que ele fosse reconhecido globalmente como um hotspot de biodiversidade (Myers et al. 2000). A biodiversidade do Cerrado é estimada em um terço da fauna e flora brasileira e $5 \%$ da mundial. Além disso, do total aproximado de 10 mil espécies de plantas do Cerrado, cerca de 44\% são endêmicas (Lamoreux et al. 2006). No entanto, é particularmente notável que o clima do Cerrado, com estações bem definidas e um período seco marcante, exerça influência sobre a comunidade epifítica, levando à ocorrência de um número relativamente menor de epífitas quando comparado ao das áreas de Florestas Úmidas (Breier 2005, Kersten 2006).

Esta pesquisa avaliou a diversidade, abundância e estratificação vertical das epífitas vasculares na
Estação Ecológica de Jataí (EEJ), uma área de Cerrado no Sudeste brasileiro, a fim de gerar informações sobre essa sinúsia nesse tipo de ambiente, contribuindo, assim, para a caracterização e conservação das epífitas vasculares em áreas de Cerrado no Brasil.

\section{Material e métodos}

O estudo foi realizado na Estação Ecológica de Jataí $\left(21^{\circ} 30^{\prime}-21^{\circ} 40^{\prime}\right.$ S e $\left.47^{\circ} 40^{\prime}-47^{\circ} 50^{\prime} \mathrm{O}\right)$, SP, Brasil, (figura 1). Situada no bioma Cerrado e com altitudes entre 515 e $835 \mathrm{~m}$, a EEJ tem área de 9.021 ha, apresentando formações campestres (Campo sujo, Campo Limpo), formações savânicas (Cerrado stricto sensu) e formações florestais (Cerradão, Mata de Galeria), sendo essas últimas as mais propícias para o desenvolvimento da comunidade epifítica herbácea vascular (Toppa 2004).

O clima da EEJ é predominante tropical-quentesubúmido (Aw) caracterizado por forte sazonalidade das chuvas e estabilidade da temperatura média diária (Setzer 1966). A precipitação média anual estimada é de $1.400 \mathrm{~mm}$, com média mensal variando de 0 - $460 \mathrm{~mm}$, e temperatura média de $22{ }^{\circ} \mathrm{C}(11,6$ $36,2^{\circ} \mathrm{C}$ ) (Castro et al. 1994). Também importantes na composição fitofisionômica do Cerrado são os fatores pedológicos, como a toxidez por alumínio, e pirogênicos, como incêndios localizados, que têm ação direta sobre a biota (Toppa 2004). Esses fatores afetam a ocorrência das espécies arbóreas (forófitos) que têm relação direta com a ocorrência das epífitas vasculares (Barthlott et al. 2001).

O trabalho de campo foi realizado entre outubro de 2009 e outubro de 2011. A diversidade de epífitas vasculares foi avaliada por metodologia assistemática (caminhadas aleatórias) ao longo da Unidade de Conservação, cobrindo uma área amostral de aproximadamente 5 ha, e a abundância das espécies foi obtida pela amostragem de 70 forófitos com DAP $\geq 30 \mathrm{~cm}$, utilizando-se para tal o método de ponto quadrante. A presença/ausência e a abundância de espécies epifíticas vasculares nos forófitos foram registradas em cinco zonas de acordo com Johansson (1974) e ter Steege \& Cornelissen (1989): Z1 - Fuste baixo; Z2 - Fuste médio; Z3 - Fuste alto; Z4 - Copa interna; Z5 - Copa externa. As epífitas foram distribuídas em três tipos ecológicos (Acebey et al. 2003, Krömer \& Kessler 2006, Krömer et al. 2007): hemiepífitas; epífitas generalistas (registradas em três ou mais zonas); e epífitas especialistas (registradas em duas ou três zonas contínuas); esse último tipo foi dividido em epífitas de copa (>90\% dos indivíduos 


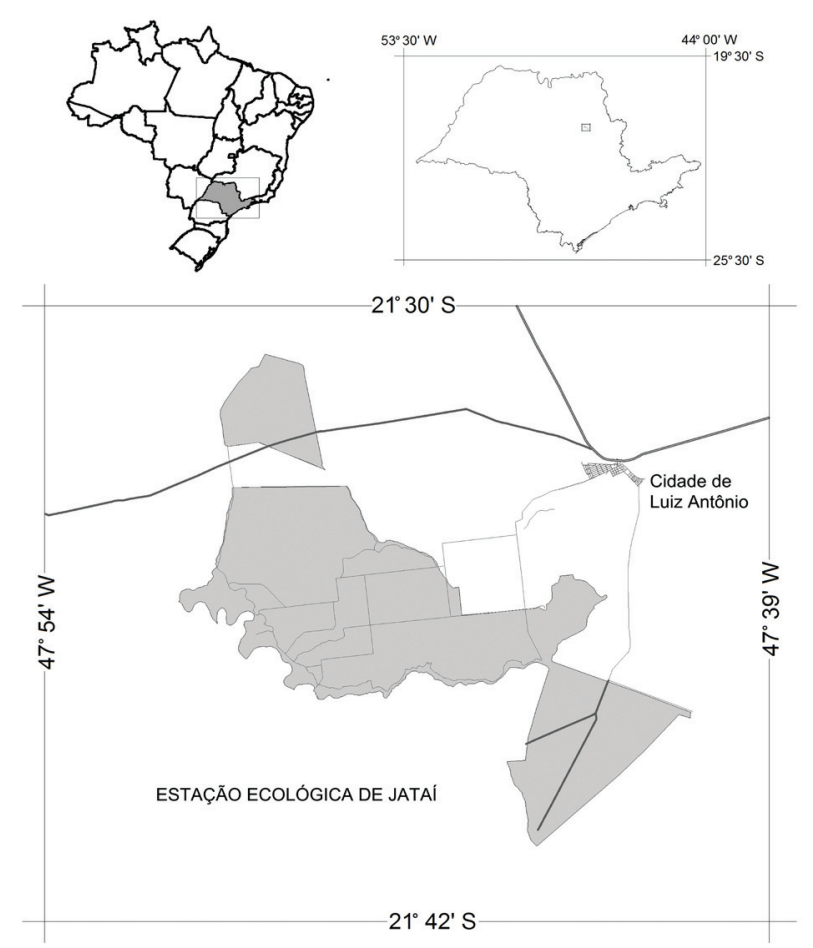

Figura 1. Localização da Estação Ecológica de Jataí, SP, Brasil.

Figure 1. Location of Jataí Ecological Station, São Paulo State, Brazil.

registrados nas Z4-Z5) e epífitas de fuste ou de sombra ( $>90 \%$ dos indivíduos registrados nas Z1-Z3) (figura 2). Além disso, as espécies epifíticas foram classificadas em categorias ecológicas, de acordo com sua relação com o forófito, a saber: holoepífitos característicos, holoepífitos facultativos, holoepífitos acidentais, hemiepífitos primários, hemiepífitos secundários (Benzing 1990, Kersten \& Silva 2001).

O material testemunha das epífitas vasculares foi processado e depositado no Herbário da Universidade Federal de São Carlos, campus São Carlos, SP. A identificação foi feita com base em bibliografia especializada para cada família vegetal amostrada, por comparação com material previamente identificado e por consulta a especialistas. Os nomes das espécies foram verificados nos sites especializados (www. tropicos.org e/ou www.kew.org.uk/epic) e em publicações taxonômicas, utilizando-se as abreviaturas dos autores sugeridas por Brummitt \& Powell (1992).

Para calcular a diversidade de espécies epifíticas vasculares da Estação Ecológica de Jataí foram utilizados os índices de Shannon-Weaver (H') e Simpson $(1 / \lambda)$, e para avaliar a partição dos indivíduos entre as espécies foi utilizada a Equidade de Pielou (J) (Magurran 1988). Para testar as possíveis diferenças entre a distribuição vertical das epífitas sobre os forófitos foram aplicados os testes $t$ de Student e de Kolmogorov-Smirnov (Magurran 1988). Para ilustrar a distribuição das abundâncias das espécies epífitas vasculares entre as diferentes zonas foi elaborado o diagrama de Whittaker (Whittaker 1967) com base nos valores de importância epifítica. Para testar a preferências das espécies epifíticas por zonas de fuste ou de copa, foi utilizada uma tabela de contingência $(2 \times 2)$, aplicando o teste Exato de Fisher, recomendado por Zar (1999). A tabela de categorias foi determinada pela presença e ausência de cada espécie nos habitats fuste e copa (Acebey et al. 2003, Krömer et al. 2007); e o padrão de distribuição dos indivíduos e das espécies entre os tipos ecológicos - hemiepífitas, epífitas generalistas, epífitas de fuste e epífitas de copa - foi comparado pelo teste G.

\section{Resultados}

No total, 1.574 epífitas vasculares pertencentes a 29 espécies, 20 gêneros e a sete famílias foram encontradas na Estação Ecológica de Jataí (tabela 1). Todas as espécies encontradas no levantamento florístico também foram registradas, pelo menos uma vez, na análise quantitativa. A diversidade de espécies epífitas vascular na área foi de 2.66 segundo o índice de diversidade de Shannon-Weaver $\left(H^{\prime}\right)$ e de 0,91 segundo o índice de Simpson $(1 / \lambda)$. A partição entre as espécies epífitas apontada pela Equidade de Pielou (J) foi de 0,79 .

Adistribuição das espécies epifíticas nas categorias ecológicas de relação com o forófito evidenciou o predomínio dos holoepífitos característicos com 27 espécies (93\%), os holoepífitos facultativos foram representados por uma espécie $(3,5 \%)$ e os hemiepífitos secundários, por uma espécie $(3,5 \%)$. Não foram registrados holoepífitos acidentais e hemiepífitos primários.

As Angiospermas foram representadas por 23 espécies e as Samambaias por seis espécies. As famílias com maior diversidade foram Bromeliaceae e Orchidaceae (oito espécies cada), Polypodiaceae (seis spp.), Piperaceae (três spp.), Cactaceae (duas spp.). Araceae e Aspleniaceae apresentaram uma espécie cada. Bromeliaceae apresentou o maior número de indivíduos epifíticos, seguida pela família Polypodiaceae com a segunda maior abundância (figura 3a). Orchidaceae, uma das famílias mais ricas na área (figura 3b), teve a quarta maior abundância. A família Cactaceae, embora com apenas duas espécies, apresentou a terceira maior abundância. 
Microgramma squamulosa (Polypodiaceae) foi a espécie mais abundante da área de estudo (tabela 1), seguida por Tillandsia stricta (Bromeliaceae), Rhipsalis baccifera (Cactaceae) e Pleopeltis pleopeltifolia (Polypodiaceae). A família Orchidaceae apresentou maior número de espécies "raras" na área de estudo; das oito espécies dessa família, seis apresentaram IVI $<1,0$ (tabela 1). Quanto ao registro das espécies epifíticas vasculares sobre os forófitos, Microgramma squamulosa e Tillandsia stricta ocorreram em mais de $71 \%$ dos forófitos (tabela 1), Pleopeltis pleopeltifolia ocorreu em $60 \%$ desses e duas Cactaceae: Epiphyllum phyllanthus e Rhipsalis baccifera, estiveram presentes em 55,7 e $52,9 \%$ dos forófitos, respectivamente.

A estratificação vertical das epífitas vasculares revelou maior número de espécies nas zonas intermediárias. As zonas Z2 e Z3 apresentaram 22 espécies epifíticas cada (figura 3a), na Z4 foram registradas 21 espécies, e nas Z1 e Z5 ocorreram 15 e oito espécies, respectivamente. A família Piperaceae dominou a ocorrência na $\mathrm{Z1}$, tendo o número de indivíduos reduzido gradativamente nas Z2, Z3 e Z4 (figura $3 b$ ). Cactaceae foi mais abundante nas zonas intermediárias, especialmente nas Z3 e Z2. Esse mesmo padrão foi observado para Orchidaceae, que apesar de apresentar o mesmo número de espécies entre Z2-Z4 (figura 3a), foi mais abundante, respectivamente nas zonas Z4, Z3, Z1 e Z2. A família Polypodiaceae foi registrada em todas as cinco zonas, sendo dominante na Z4. As Bromeliaceae também foram registradas em todas as zonas, dominando a Z5 com mais de 70\% dos indivíduos nessa zona (figura $3 b$ ); mas foram abundantes também nas Z4,Z3 eZ2, e apresentaram um número menor de registros na Z1. As famílias Araceae e Aspleniaceae foram registradas apenas na Z4 e Z1, respectivamente.
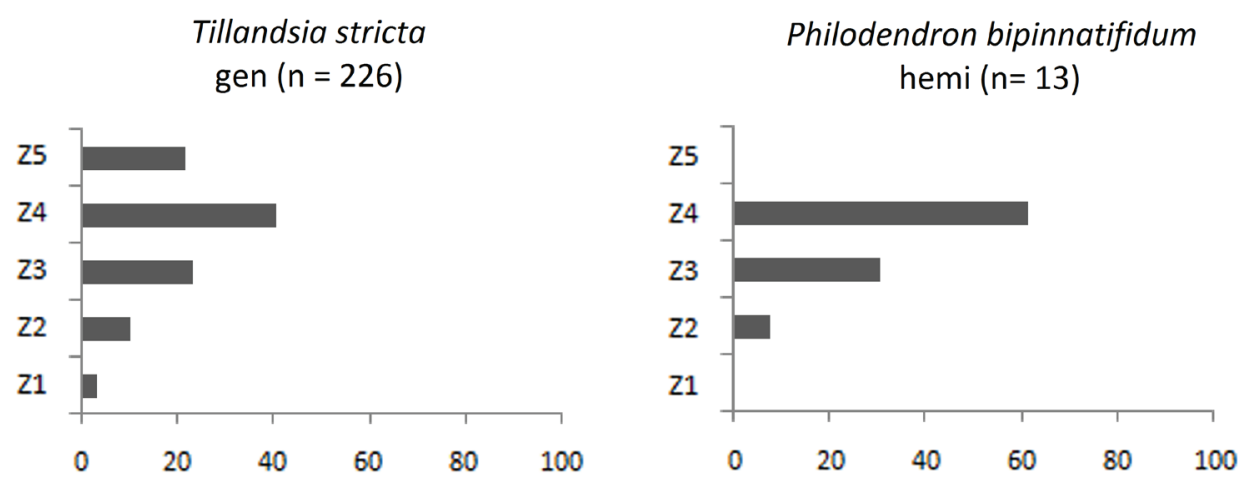

Campyloneurum nitidum trunk $(n=21)$

Pleopeltis squalida capony $(\mathrm{n}=22)$

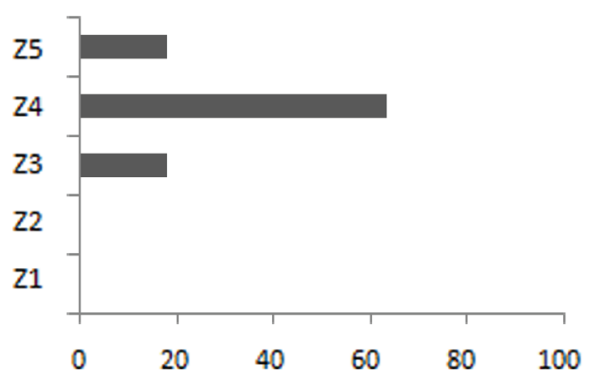

registros (\%)

Figura 2. Percentual de registros de quatro espécies epifíticas vasculares, definida como presença/ausência da espécie em área de Cerrado do Sudeste do Brasil, selecionadas nas cinco zonas de altura. hemi: hemiepífitas; gen: epífitas generalistas; trunk: epífitas de fuste ou de sombra; canopy: epífitas de copa.

Figure 2. Percentages of records of four selected vascular epiphytes species, defined as the presence/absence of the species in the Cerrado area of southeastern Brazil, in the five height zones. hemi: hemiepiphyte; gen: habitat generalist; trunk: trunk epiphyte; canopy: canopy epiphytes. 
Tabela 1. Diversidade de epífitas vasculares encontradas na Estação Ecológica de Jataí, área de Cerrado no Sudeste do Brasil. Freq.: frequência; No. For.: número de forófitos; IVI: índice do valor de importância. Classificação nas categorias ecológicas (CE) - HLC: holoepífito característico; HLF: holoepífito facultativo; HLA: holoepífito acidental; HMP: hemiepífito primário; HMS: hemiepífito secundário.

Table 1. Diversity of vascular epiphytes found in Estação Ecológica de Jataí, Southeast Brazilian Cerrado area. . Freq.: frequency; No. For.: number of host trees; IVI: importance value index. Ecological categories (CE) - HLC: characteristic holoepiphyte; HLF: facultative holoepiphyte; HLA: accidental holoepiphyte; HMP: primary hemiepiphyte; HMS: secondary hemiepiphyte.

\begin{tabular}{|c|c|c|c|c|}
\hline Família / Espécies & Freq. & No. For. & IVI & $\mathrm{CE}$ \\
\hline \multicolumn{5}{|l|}{ Araceae } \\
\hline Philodendron bipinnatifidum Schott ex Endl. & 7,1 & 5,0 & 0,83 & HMS \\
\hline \multicolumn{5}{|l|}{ Aspleniaceae } \\
\hline Asplenium serratum $\mathrm{L}$. & 2,9 & 2,0 & 0,25 & HLC \\
\hline \multicolumn{5}{|l|}{ Bromeliaceae } \\
\hline Acanthostachys strobilacea (Schult. f.) Klotzsch & 28,6 & 20,0 & 2,22 & HLC \\
\hline Aechmea bromeliifolia (Rudge) Baker & 17,1 & 12,0 & 1,08 & HLC \\
\hline Aechmea nudicaulis (L.) Griseb. & 1,4 & 1,0 & 0,06 & HLC \\
\hline Billbergia distachia (Vell.) Mez & 2,9 & 2,0 & 0,19 & HLC \\
\hline Billbergia zebrina (Herb.) Lindl. & 14,3 & 10,0 & 1,27 & HLC \\
\hline Tillandsia recurvata (L.) L. & 35,7 & 25,0 & 7,69 & HLC \\
\hline Tillandsia stricta Sol. ex Sims & 71,4 & 50,0 & 14,36 & HLC \\
\hline Tillandsia tricholepis Baker & 14,3 & 10,0 & 2,86 & HLC \\
\hline \multicolumn{5}{|l|}{ Cactaceae } \\
\hline Epiphyllum phyllanthus (L.) Haw. & 55,7 & 39,0 & 7,50 & HLC \\
\hline Rhipsalis baccifera (J.S. Muell.) Stearn & 52,9 & 37,0 & 10,99 & HLC \\
\hline \multicolumn{5}{|l|}{ Orchidaceae } \\
\hline Catasetum fimbriatun (C. Morren) Lindl. & 4,3 & 3,0 & 0,44 & HLC \\
\hline Coppensia varicosa (Lindl. \& Paxton) Campacci & 38,6 & 27,0 & 5,65 & HLC \\
\hline Cyrtopodium sp. & 1,4 & 1,0 & 0,13 & HLC \\
\hline Epidendrum pseudodifforme Hoehne \& Schltr. & 2,9 & 2,0 & 0,19 & HLC \\
\hline Rodriguezia decora Rchb. f. & 4,3 & 3,0 & 0,95 & HLC \\
\hline Polystachya estrellensis Rchb. f. & 25,7 & 18,0 & 4,38 & HLC \\
\hline Laelia gloriosa (Rchb. f.) L.O. Williams. & 1,4 & 1,0 & 0,19 & HLC \\
\hline Sophronitis cernua Lindl. & 1,4 & 1,0 & 0,19 & HLC \\
\hline \multicolumn{5}{|l|}{ Piperaceae } \\
\hline Peperomia cyclophylla Miq. & 30,0 & 21,0 & 5,91 & HLC \\
\hline Peperomia glabella (Sw.) A. Dietr. & 4,3 & 3,0 & 0,76 & HLC \\
\hline Peperomia pereskiifolia (Jacq.) Kunth & 20,0 & 14,0 & 4,45 & HLF \\
\hline \multicolumn{5}{|l|}{ Polypodiaceae } \\
\hline Campyloneurum nitidum (Kaulf.) C. Presl & 7,1 & 5,0 & 1,33 & HLC \\
\hline Microgramma lindbergii (Mett. ex Kuhn) de la Sota & 1,4 & 1,0 & 0,13 & HLC \\
\hline Microgramma squamulosa (Kaulf.) de la Sota & 71,4 & 50,0 & 14,49 & HLC \\
\hline Pleopeltis pleopeltifolia (Raddi) Alston & 60,0 & 42,0 & 10,04 & HLC \\
\hline Pleopeltis squalida (Vell.) de la Sota & 8,6 & 6,0 & 1,40 & HLC \\
\hline Serpocaulon latipes (Langsd. \& L. Fisch.) A.R. Sm. & 1,4 & 1,0 & 0,06 & HLC \\
\hline
\end{tabular}


As quatro espécies mais importantes da área de estudo - Microgramma squamulosa, Tillandsia stricta, Rhipsalis baccifera e Pleopeltis pleopeltifolia (tabela 1) foram as únicas registradas em todas as zonas analisadas (figura 4). As zonas Z1 e Z5 apresentaram a maior dominância de espécies, Peperomia cyclophylla foi predominante na $\mathrm{Z} 1$ e duas Tillandsia (T. recurvata e T. stricta) dominaram a Z5. As 13 espécies raras $(I V I<1)$ foram registradas nas zonas intermediárias (Z2-Z4), com exceção de Billbergia distachia e Catasetum fimbriatum que também foram registradas na Z1. Somente as zonas Z2 e Z4 apresentaram espécies exclusivas. Aechmea nudicaulis, Cyrtopodium sp. e Serpocaulon latipes, na Z2, e Epidendrum pseudodifforme, Microgramma lindbergii e Laelia gloriosa, na Z4 (figura 4). A distribuição das abundâncias das espécies epifíticas nas zonas (figura 4) seguiu o modelo LogSérie $(P<0,001)$, exceto Z1 que teve distribuição LogNormal, e Z5, que apresentou distribuição geométrica $(P<0,05)$.

$\mathrm{O}$ teste de Kolmogorov-Smirnov revelou uma diferença significativa entre as abundâncias

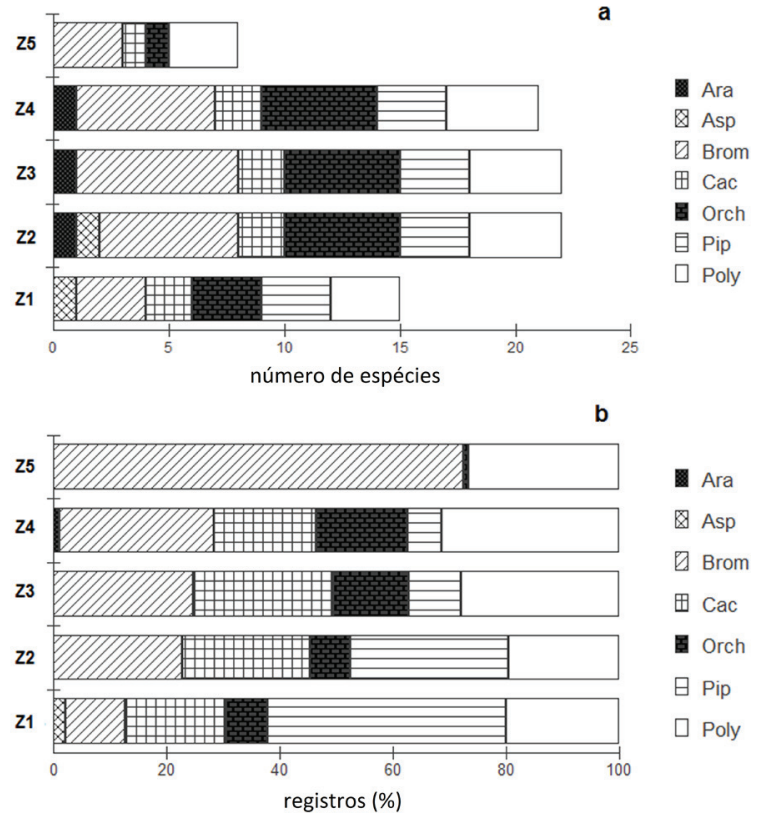

Figura 3. Riqueza de espécies e percentual de indivíduos nas famílias epifíticas em área de Cerrado no sudeste brasileiro nas cinco zonas de altura. Ara: Araceae; Asp: Aspleniaceae; Brom: Bromeliaceae; Cac: Cactaceae; Orch: Orchidaceae; Pip: Piperaceae; Poly: Polypodiaceae.

Figure 3. Species richness and percentages of records in the epiphytic families in southeast Brazilian Cerrado area in the five height zones. Ara: Araceae; Asp: Aspleniaceae; Brom: Bromeliaceae; Cac: Cactaceae; Orch: Orchidaceae; Pip: Piperaceae; Poly: Polypodiaceae. das espécies epifíticas que ocorreram em: Z1 - Z3 $(P<0,05), \mathrm{Z} 1$ - Z4 $(P<0,01), \mathrm{Z} 2-\mathrm{Z} 5(P<0,005)$, Z3 - Z5 $(P<0,005)$ e entre as zonas Z4 - Z5 $(P<0,005)$. As demais zonas não foram significativamente diferentes. O teste $t$ aplicado a diversidade revelou Z1 significativamente diferente de todas as outras zonas $(P<0,05)$ e esse mesmo resultado foi registrado para a zona Z5 $(P<0,001)$.

O registro das epífitas vasculares em tipos ecológicos (tabela 2) na área de Cerrado estudada revelou maior número de espécies especialistas (55\%), seguidas pelas espécies generalistas (41\%) e hemiepífitas (4\%). Dentre as especialistas, 69\% são epífitas de copa e 31\% epífitas de fuste (epífitas de sombra). No total, cerca de $65 \%$ das epífitas de copa foram significativamente mais frequentes no seu habitat preferencial (teste Exato de Fisher, $P<0,05$ ); no entanto, nenhuma das epífitas de fuste apresentou preferência significativa. Entre as epífitas generalistas, mais de $65 \%$ não apresentaram diferença significativa entre as zonas de fuste ou de copa (tabela 2).

Todas as espécies de Piperaceae e Cactaceae foram generalistas. Bromeliaceae apresentou 50\% das espécies generalistas, $38 \%$ especialistas de copa e $12 \%$ especialistas de fuste. Orchidaceae teve mais de $60 \%$ das espécies como especialistas de copa, $26 \%$ como generalistas e $12 \%$ como especialistas de fuste. A maioria das espécies de Polypodiaceae foi representada por especialistas de copa $(50 \%)$ ou especialistas de fuste (33\%); Aspleniaceae (1 sp.) foi especialista de fuste e Araceae (1 sp.) foi hemiepífita.

\section{Discussão}

A comunidade epifítica vascular na área de Cerrado estudada apresentou uma riqueza de espécies baixa, sobretudo quando comparada com comunidades em florestas úmidas no Brasil (e.g. Hertel 1950, Dittrich et al. 1999, Giongo \& Waechter 2004, Kersten 2006). No entanto, estudos conduzidos em áreas com acentuada sazonalidade climática ou ocupadas por vegetação de clima seco tendem a apresentar baixa riqueza epifítica (Yeaton \& Gladstone 1982, Werneck \& Espírito-Santo 2001, Breier 2005, Ishara et al. 2008). Portanto, a baixa riqueza epifítica observada na área estudada pode estar relacionada à condição de sazonalidade climática e ao tipo de vegetação característico em áreas de Cerrado (vegetação aberta). Isso submete a comunidade epifítica a uma condição de alta luminosidade e de baixa disponibilidade hídrica, especialmente no período de estiagem, 
fatores conhecidos por serem limitantes à riqueza e abundância de epífitas vasculares (ter Steege \& Cornelissen 1989, Benzing 1990).

O predomínio de holoepífitos característicos observado na área era esperado e tem sido comum em áreas de Cerrado (Breier 2005, Ishara et al. 2008), em áreas de Floresta Estacional Semidecidual (Dislich \& Mantovani 1998, Kersten \& Kuniyoshi 2009, Bataghin et al. 2010), em outras formações florestais como a Floresta Ombrófila Mista (Dittrich et al. 1999, Kersten 2006) e áreas de restinga (Waechter 1992, Kersten \& Silva 2001).

A maior riqueza das famílias Bromeliaceae e Orchidaceae pode estar relacionada à presença de características adaptativas, como os tanques de bromélias e os órgãos suculentos de reserva nas orquídeas, que são bem adaptadas a ambientes secos e com maior exposição à luz (Smith 1986). Barthlott et al. (2001), encontraram maior diversidade de espécies de bromélias em floresta secundária do que em floresta primária, e afirmaram que a maior resistência dessas a ambientes secos e de maior luminosidade poderia ser responsável por tais resultados. Além disso, Orchidaceae e Bromeliaceae estão entre as três famílias de epífitas mais abundantes

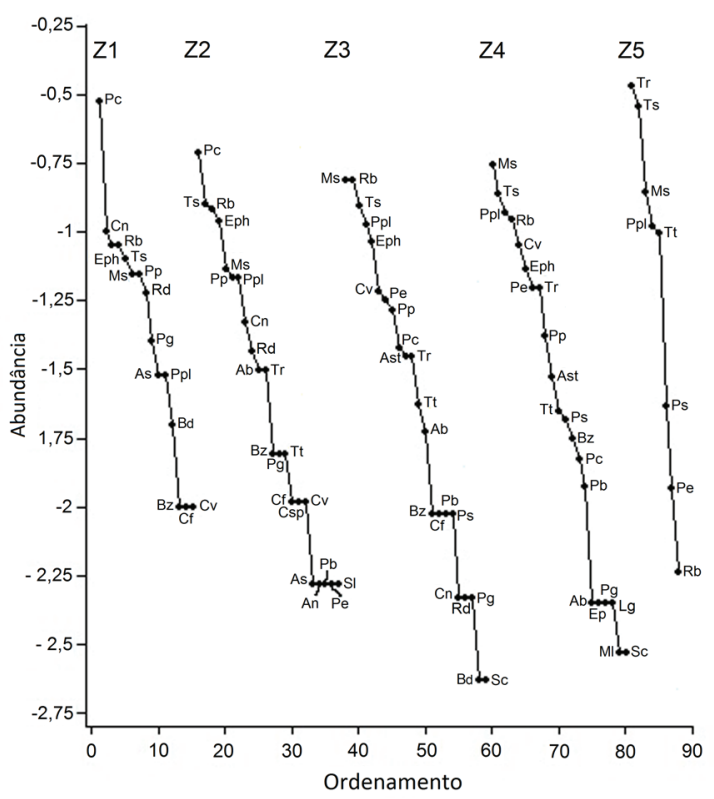

Figura 4. Diagrama de distribuição das abundâncias das epífitas vasculares nas zonas de altura em área de Cerrado no sudeste do Brasil. Abreviaturas dos nomes das espécies nas curvas, de acordo com a tabela 2 .

Figure 4. Diagram of distribution of abundances of vascular epiphytes in the height zones in southeastern Brazilian cerrado area. Abbreviation of species names on the curves according to table 2. mundialmente (Benzing 1990). É notável a presença das Bromeliaceae, tanto pela riqueza quanto pela abundância (figura $3 \mathrm{a}, \mathrm{b}$ ), e isso pode ter relação também com endemismo neotropical dessa família (Dislich \& Mantovani 1998).

A família Orchidaceae, que apresenta cerca de 20.000 espécies, e é a mais rica em epífitas, tanto mundialmente (Kress 1986, Benzing 1990) quanto no neotrópico (Gentry \& Dodson 1987), contribuiu com oito espécies, sendo uma das mais ricas desse estudo, embora o clima seco da região possa ter limitado a abundância dessa família na EEJ. O reflexo da ação das condições climáticas sobre as Orchidaceae é evidenciado pelo baixo IVI $(<1,0)$ de seis das oito espécies de orquídeas registradas (tabela 1$)$.

A influência favorável do clima do Cerrado sobre as famílias Polypodiaceae e Cactaceae também ficou evidente. A família Polypodiaceae, que apresentou seis espécies e foi a segunda mais abundante (figura 3b), teve duas espécies (Microgramma squamulosa e Pleopeltis pleopeltifolia) como dominantes. Essas espécies apresentam, além da plasticidade fenotípica de seus esporos, a capacidade de mantê-los viáveis por longos períodos (Tryon 1970), característica fundamental à sobrevivência em situações de sazonalidade climática. A presença de adaptações climáticas (p. ex. a via de metabolismo CAM e a presença de caules suculentos) também ocorre nas espécies da família Cactaceae (Hunt et al. 2006), que apesar de apresentar apenas duas espécies, teve grande abundância (figura $3 a, b$ ).

A presença de mecanismos ou adaptações às condições climáticas influencia no sucesso de espécies que, mesmo em ambiente pouco favorável ao epifitismo, apresentaram grande distribuição nos forófitos, alta abundância e, consequentemente, IVI maior (tabela 1).

A estratificação vertical das epífitas vasculares é determinada por vários fatores microclimáticos, como a intensidade de luz, velocidade do vento e a umidade relativa do ar (Benzing 1995, Nieder et al. 1999, Barthlott et al. 2001). Notadamente, a temperatura e a intensidade luminosa aumentam do solo para o dossel, enquanto a umidade tende a seguir o sentido inverso (Parker 1995, Freiberg 1997). Apesar disso, na base da copa, o acúmulo de matéria orgânica serve como suplemento de água e nutrientes para as epífitas vasculares (Krömer et al. 2007).

No presente estudo, a maior riqueza de epífitas foi observada nas zonas intermediárias - Z2 a Z4 
(figura 3a), nas quais existe um equilíbrio entre o nível de luminosidade e a disponibilidade hídrica, além do acúmulo de matéria orgânica que fornece um ambiente propício para a ocorrência de epífitas vasculares. O registro da maior riqueza nas zonas $\mathrm{Z} 2$ e Z3 evidencia a importância da umidade para o sucesso de espécies epifíticas na área de Cerrado. A maior riqueza das zonas intermediarias observada na área corrobora os resultados obtidos por ter Steege \& Cornelissen (1989), Freiberg (1996), Nieder et al. 1999, Acebey \& Krömer (2001), Krömer et al. (2007).

$\mathrm{Na} Z 5$, o reduzido número de espécies registradas indica que as acentuadas variações microclimáticas da copa externa, caracterizadas pelos prolongados

Tabela 2. Registro das espécies de epífitas vasculares nos tipos ecológicos em área de Cerrado no sudeste do Brasil. Os valores de $P$ obtidos no teste Exato de Fisher $(2 \times 2)$ apresentam preferência de habitat $(P<0,05)$; ns: não significativo. hemi: hemiepífitas; gen: epífitas generalistas; canopy: epífitas de copa; trunk: epífitas de fuste ou de sombra.

Table 2. Species records of vascular epiphytes in ecological types in southeast Brazilian Cerrado area. $P$ values obtained from Fisher exact test $(2 \times 2)$ present habitat preference $(P<0.05)$; ns: not significant. hemi: hemiepiphyte; gen: habitat generalist; canopy: canopy epiphytes; trunk: trunk epiphyte.

\begin{tabular}{|c|c|c|c|c|c|c|c|c|}
\hline Espécies & Leg. & Reg. & $\begin{array}{l}\text { Pres. no } \\
\text { fuste }\end{array}$ & $\begin{array}{l}\text { Aus. no } \\
\text { fuste }\end{array}$ & $\begin{array}{c}\text { Pres. na } \\
\text { copa }\end{array}$ & $\begin{array}{c}\text { Aus. na } \\
\text { copa }\end{array}$ & $P$ & $\begin{array}{l}\text { Tipo } \\
\text { Ecol. }\end{array}$ \\
\hline Philodendron bipinnatifidum & $\mathrm{Pb}$ & 13 & 1 & 69 & 5 & 65 & ns & hemi \\
\hline Asplenium serratum & As & 4 & 1 & 69 & 0 & 70 & ns & trunk \\
\hline Acanthostachys strobilacea & Ast & 35 & 0 & 70 & 20 & 50 & $<0,001$ & canopy \\
\hline Aechmea bromeliifolia & $\mathrm{Ab}$ & 17 & 5 & 65 & 7 & 63 & ns & gen \\
\hline Aechmea nudicaulis & An & 1 & 1 & 69 & 0 & 70 & ns & trunk \\
\hline Billbergia distachia & $\mathrm{Bd}$ & 3 & 1 & 69 & 1 & 69 & $\mathrm{~ns}$ & gen \\
\hline Billbergia zebrina & $\mathrm{Bz}$ & 20 & 3 & 67 & 8 & 62 & ns & gen \\
\hline Tillandsia recurvata & $\operatorname{Tr}$ & 121 & 6 & 64 & 25 & 45 & $<0,001$ & canopy \\
\hline Tillandsia stricta & Ts & 226 & 23 & 47 & 49 & 21 & $<0,001$ & gen \\
\hline Tillandsia tricholepis & $\mathrm{Tt}$ & 45 & 3 & 67 & 10 & 60 & $<0,05$ & canopy \\
\hline Epiphyllum phyllanthus & Eph & 118 & 15 & 55 & 34 & 36 & $<0,001$ & gen \\
\hline Rhipsalis baccifera & $\mathrm{Rb}$ & 173 & 22 & 48 & 35 & 35 & $<0,05$ & gen \\
\hline Catasetum fimbriatum & $\mathrm{Cf}$ & 7 & 2 & 68 & 2 & 68 & ns & gen \\
\hline Coppensia varicosa & $\mathrm{Cv}$ & 89 & 2 & 68 & 27 & 43 & $<0,001$ & canopy \\
\hline Cyrtopodium sp. & Csp & 2 & 1 & 69 & 0 & 70 & ns & trunk \\
\hline Epidendrum pseudodifforme & Ep & 3 & 0 & 70 & 2 & 68 & $\mathrm{~ns}$ & canopy \\
\hline Rodriguezia decora & $\mathrm{Rd}$ & 15 & 3 & 67 & 2 & 68 & ns & gen \\
\hline Polystachya estrellensis & $\mathrm{Pe}$ & 69 & 1 & 69 & 18 & 52 & $<0,001$ & canopy \\
\hline Laelia gloriosa & $\operatorname{Lg}$ & 3 & 0 & 70 & 1 & 69 & ns & canopy \\
\hline Sophronitis cernua & $\mathrm{Sc}$ & 3 & 0 & 70 & 1 & 69 & ns & canopy \\
\hline Peperomia cyclophylla & $\mathrm{Pc}$ & 93 & 19 & 51 & 14 & 56 & $\mathrm{~ns}$ & gen \\
\hline Peperomia glabella & $\mathrm{Pg}$ & 12 & 3 & 67 & 1 & 69 & ns & gen \\
\hline Peperomia pereskiifolia & $\mathrm{Pp}$ & 70 & 9 & 61 & 13 & 57 & ns & gen \\
\hline Campyloneurum nitidum & $\mathrm{Cn}$ & 21 & 5 & 65 & 2 & 68 & ns & trunk \\
\hline Microgramma lindbergii & Ml & 2 & 0 & 70 & 1 & 69 & ns & canopy \\
\hline Microgramma squamulosa & Ms & 228 & 12 & 58 & 49 & 21 & $<0,001$ & canopy \\
\hline Pleopeltis pleopeltifolia & Ppl & 158 & 10 & 60 & 42 & 28 & $<0,001$ & gen \\
\hline Pleopeltis squalida & Ps & 22 & 0 & 70 & 6 & 64 & $<0,05$ & canopy \\
\hline Serpocaulon latipes & Sl & 1 & 1 & 69 & 0 & 70 & $\mathrm{~ns}$ & trunk \\
\hline
\end{tabular}


períodos de baixa umidade relativa do ar, altas temperaturas e luminosidade, e maior intensidade do vento (Benzing 1995, Krömer et al. 2007), exercem forte efeito sobre a comunidade epifítica vascular. Em adição, as espécies registradas na copa externa - sobretudo as Tillandsia (figura 4) apresentam mecanismos ou adaptações que permitem a ocorrência em locais ou ambientes pouco propícios ao epifitismo (Bonnet \& Queiroz 2006, Tryon 1970, Hunt et al. 2006).

A composição específica da comunidade epifítica no gradiente vertical, revelou as famílias Piperaceae e Bromeliaceae como mais abundantes, respectivamente, nas zonas inferiores $(Z 1)$ e nas zonas superiores (Z5), essa última dominada por espécies de Tillandsia (Bromeliaceae). Além disso, foi registrado maior número de indivíduos de Orchidaceae, Cactaceae e Polypodiaceae nas zonas intermediárias (figura 3b), representando um arranjo similar a distribuição taxonômica que foi observada nos estudos de ter Steege \& Cornelissen (1989), Acebey \& Krömer (2001) e Krömer et al. (2007).

O modelo de distribuição da abundância das epífitas (Whittaker 1967) nas zonas propostas por Johansson (1974) e ter Steege \& Cornelissen (1989), apontou comportamento semelhante nas comunidades epífitas das zonas Z2-Z4 (figura 4), pois a distribuição das espécies na curva indica maior diversidade e maior

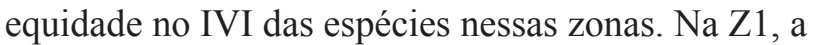
distribuição dos pontos na curva mostrou um número maior de espécies de abundância intermediária, poucas espécies dominantes e um pequeno número de espécies de baixa abundância. Já na Z5 o decréscimo da curva mostrou a dominância de poucas espécies, com uma redução rápida e quase constante da abundância para as demais espécies (figura 4). De forma geral, a distribuição na área de Cerrado estudada reflete uma comunidade relativamente simples, com espécies que competem ou resistem de modo forte e hierárquico sob pressão de um ou poucos fatores dominantes (Whittaker 1967). Além disso, os testes Te Kolmogorov-Smirnov revelaram que tanto $\mathrm{Z} 1$ quanto Z5 apresentaram diferença significativa no número de registros e na diversidade epifítica vascular em relação às demais zonas estudadas.

Um importante resultado obtido foi a identificação de um número maior de espécies especialistas (cerca de 55\%), sobretudo epífitas de copa. A razão para esse maior número de especialistas no dossel, presumivelmente, envolve uma combinação de mecanismos e adaptações das espécies, além de fatores climáticos e da disponibilidade de substrato na parte superior das árvores hospedeiras (Krömer et al. 2007, Poltz \& Zotz 2011). Cabe destacar, também, as epífitas de sombra ou de fuste que somaram mais de $30 \%$ das espécies especialistas. Isso possivelmente está relacionado com o fato de o sub-bosque apresentar condições de menor intensidade luminosa, maior umidade relativa do ar e menor incidência de ventos do que nas zonas de dossel (Parker 1995, Krömer et al. 2007).

Considerando, ainda, os tipos ecológicos, dentre as espécies de Cactaceae, Bromeliaceae e Piperaceae mais de $80 \%$ são generalistas, sendo ecologicamente flexíveis e podendo ocorrer desde as zonas próximas ao solo até o dossel dos forófitos. Distribuição semelhante a que foi observada por Acebey \& Krömer (2001) e Krömer et al. (2007). Entre as espécies generalistas, cerca de $30 \%$ apresentaram preferência significativa (tabela 2) pela copa ou pelo fuste. Dentre as especialistas, somente as epífitas de copa (65\% dessas) apresentaram preferência significativa (tabela 2) segundo o teste Exato de Fisher, o que indica alta especificidade das epífitas que ocorrem no dossel na área de Cerrado. Epífitas de copa foram representadas principalmente por orquídeas, samambaias e algumas bromélias, as quais apresentam adaptações para o estresse hídrico, tais como pseudobulbos, caules ou folhas suculentas, roseta em forma de tanque ou são poiquiloídricas (Benzing 1990), como por exemplo, A. strobilacea (figura 2), T. recurvata, P. estrellensis e $P$. squalida. Embora em fitofisionomia distinta à estudada nesta pesquisa, os dados de Krömer et al. 2007 revelaram orquídeas, samambaias e bromélias como espécies preferenciais na copa de duas florestas úmidas nos Andes Bolivianos. Entretanto, estudos detalhados em áreas de floresta seca são fundamentais para identificar se esse modelo de distribuição pode ser generalizado.

Araceae e Aspleniaceae apresentaram apenas uma espécie cada e um número baixo de indivíduos, o que não permite discutir a influência dessas famílias nessa classificação ecológica. No entanto, espécies de Araceae, de forma geral, apresentam características ligadas ao hemiepifitismo (Prósperi et al. 2001), e Aspleniaceae tende a apresentar espécies nos estratos inferiores da floresta (Krömer et al. 2007), já que podem sobreviver com limitado suprimento de luz (Schneider et al. 2004). Essa mesma situação foi observada na área de Cerrado aqui estudada. 
De forma geral, a diversidade epifítica encontrada é alta quando comparada a estudos realizados em áreas contínuas de Cerrado, embora possa ser considerada baixa se relacionada a florestas úmidas. A maior diversidade observada nas zonas intermediárias dos forófitos reflete a busca por uma condição climática favorável, na qual haja equilíbrio entre a disponibilidade de luz e umidade. A distribuição das abundâncias fornece indícios de uma comunidade epifítica limitada pelos fatores microclimáticos, especialmente pelo número de espécies com adaptações para resistir às mudanças nesses fatores. Mais da metade das espécies são especialistas (fuste ou copa), apontando a especificidade e limitações para a ocorrência de espécies epifíticas em áreas de Cerrado. Pesquisas futuras devem ser direcionadas ao entendimento dos mecanismos que mantém a diversidade epifítica de floresta seca, uma vez que essas informações são fundamentais para o conhecimento e conservação de epífitas vasculares nesse importante abrigo da diversidade que são as áreas de Cerrado.

\section{Agradecimentos}

Os autores agradecem a direção da Estação Ecológica de Jataí, Luiz Antonio-SP, por permitir a realização dessa pesquisa; à Fundação de Apoio Institucional - FAI/UFSCar (Projeto: 927-05) pelo apoio financeiro; à Fundação de Amparo a Pesquisa do Estado de São Paulo - FAPESP; aos Drs. Jefferson Prado e Armando Reis Tavares, do Instituto de Botânica de São Paulo, pelo auxílio na identificação de espécies; e ao Laboratório de Análise e Planejamento Ambiental (LAPA-UFSCar), por possibilitar a realização desse trabalho; Fábio de Barros agradece ao $\mathrm{CNPq}$ pela bolsa de produtividade em pesquisa recebida.

\section{Literatura citada}

Acebey, A. \& Krömer, T. 2001. Diversidad y distribución vertical de epífitas en los alrededores del campamento río Eslabón y de la laguna Chalalán, Parque Nacional Madidi, Dpto. La Paz, Bolivia. Revista de la Sociedad Boliviana de Botánica 3: 104-123.

Acebey, A., Gradstein, S.R. \& Krömer, T. 2003. Species richness and habitat diversification of bryophytes in submontane rain forest and fallows of Bolivia. Journal of Tropical Ecology 19: 9-18.

Arévalo, R. \& Betancur, J. 2006. Vertical distribution of vascular epiphytes in four forest types of the Serranía de Chiribiquete, Colombian Guayana. Selbyana 27: 175-185.
Barthlott, W., Schimit-Neuerburg, V., Nieder, J. \& Engwald, S. 2001. Diversity and abundance of vascular epiphytes: a comparison of secondary vegetation and primary montane rain forest in the Venezuelan Andes. Plant Ecology 152: 145-156.

Bataghin, F.A., Barros, F. \& Pires, J.S.R. 2010. Distribuição da comunidade de epífitas vasculares em sítios sob diferentes graus de perturbação na Floresta Nacional de Ipanema, São Paulo, Brasil. Revista Brasileira de Botânica 33: 531-542.

Benzing, D.H. 1990. Vascular epiphytes: general biology and related biota. Cambridge University Press, Cambridge.

Benzing, D.H. 1995. The physical mosaic and plant variety in forest canopies. Selbyana 16: 159-168.

Bonnet, A. \& Queiroz, M.H. 2006. Estratificação vertical de bromélias epifíticas em diferentes estádios sucessionais da Floresta Ombrófila Densa, Ilha de Santa Catarina, Santa Catarina, Brasil. Revista Brasileira de Botânica 29: 217-228.

Breier, T.B. 2005. O epifitismo vascular em florestas do sudoeste do Brasil. Tese de Doutorado, Universidade Estadual de Campinas, Campinas.

Brummitt, R.K. \& Powell, C.E. 1992. Authors of plant names. 1st ed. Royal Botanic Gardens, Kew.

Castro, L.H.R., Moreira, A.M. \& Assad, E.D. 1994. Definição e regionalização dos padrões pluviométricos dos Cerrados brasileiros. In: E.D. Assad (ed.). Chuva no Cerrado: análise e espacialização. Empresa Brasileira de Pesquisa Agropecuária, Centro de Pesquisa Agropecuária do Cerrado, Brasília, pp.13-23.

Dislich, R. \& Mantovani, W. 1998. Flora de epífitas vasculares da Reserva da Cidade Universitária "Armando de Salles Oliveira" (São Paulo, Brasil). Boletim de Botânica da Universidade de São Paulo 17: 61-83.

Dittrich, V.A.O., Kozera, C. \& Silva, S.M. 1999. Levantamento florístico de epífitos vasculares no Parque Barigui, Paraná, Brasil. Iheringia, série Botânica 52: $11-22$.

Edwards, P.J. \& Grubb, P.J. 1977. Studies of mineral cycling in a montane rain forest in New Guinea. I. The distribution of organic matter in the vegetation and soil. Journal of Ecology 65: 943-969.

Flores-Palacios, A. \& García-Franco, J.G. 2006. The relationship between tree size and epiphyte species richness: testing four different hypotheses. Journal of Biogeography 33: 323-330.

Freiberg, M. 1996. Spatial distribution of vascular epiphytes on three emergent canopy trees in French Guiana. Biotropica 28: 345-355.

Freiberg, M. 1997. Spatial and temporal pattern of temperature and humidity of a tropical premontane rain forest tree in Costa Rica. Selbyana 18: 77-84. 
Gentry, A.H. \& Dodson, C.H. 1987. Diversity and biogeography of neotropical vascular epiphytes. Annals of the Missouri Botanical Garden 74: 205-233.

Giongo, C. \& Waechter, J.L. 2004. Composição florística e estrutura comunitária de epífitos vasculares em uma floresta de galeria na Depressão Central do Rio Grande do Sul. Revista Brasileira de Botânica 27: 563-572.

Hertel, R.J.G. 1950. Contribuição à ecologia de flora epifítica da serra do mar (vertente oeste) do Paraná. Arquivos do Museu Paranaense 8: 3-63.

Hunt, D., Taylor, N. \& Charles, G. 2006, The new cactus lexicon. DH Books, Milborne Port.

Ishara, K.L., Déstro, G.F.G., Maimoni-Rodella, R.C.S. \& Yanagizawa, Y.A.N.P. 2008. Composição florística de remanescente de cerrado sensu stricto em Botucatu, SP. Revista Brasileira de Botânica 31: 575-586.

Johansson, D. 1974. Ecology of vascular epiphytes in West African rain forest. Acta Phytogeographica Suecica 59: 1-136.

Kersten, R.A. 2006. Epifitismo vascular na bacia do Alto Iguaçu, Paraná. Tese de Doutorado, Universidade Federal do Paraná, Curitiba.

Kersten, R.A. \& Kuniyoshi, Y.S. 2009. Conservação das florestas na Bacia do Alto Iguaçu, Paraná - Avaliação da comunidade de epífitas vasculares em diferentes estágios serais. Floresta 39: 51-66.

Kersten, R.A. \& Silva, S.M. 2001. Composição florística e distribuição espacial de epífitas vasculares em floresta da planície litorânea da Ilha do Mel, Paraná, Brasil. Revista Brasileira de Botânica 24: 213-226.

Kira, T. \& Yoda, K. 1989. Vertical stratification in microclimate. In: H. Lieth \& M.J.A. Werger (eds.). Ecosystems of the world. v.14b. Tropical Rain Forest ecosystems. Elsevier, Amsterdam, pp. 7-53.

Köster, N., Friedrich, K., Nieder, J. \& Barthlott, W. 2009. Conservation of epiphyte diversity in an Andean landscape transformed by human land use. Conservation Biology 23: 911-919.

Kress, J.W. 1986. A symposium: The biology of tropical epiphytes. Selbyana 9: 1-22.

Krömer, T. \& Kessler, M. 2006. Filmy ferns (Hymenophyllaceae) as high-canopy epiphytes. Ecotropica 12: 57-63

Krömer, T., Kessler, M. \& Gradstein, R.S. 2007. Vertical stratification of vascular epiphytes in submontane and montane forest of the Bolivian Andes: the importance of the understory. Plant Ecology 189: 261-278.

Lamoreux, J.F., Morrison, J.C., Ricketts, T.H., Olson, D.M., Dinerstein, E., McKnight, M.W. \& Shugart, H.H. 2006. Global tests of biodiversity concordance and the importance of endemism. Nature 440: 212-214.
Lugo, A.E. \& Scatena, F.N. 1992. Epiphytes and climate change research in the Caribbean: a proposal. Selbyana 13: $123-130$.

Magurran, A.E. 1988. Ecological diversity and its measurement. Princeton University Press, Princeton.

Myers, N., Mittermeier, R.A., Mittermeier, C.G., Fonseca, G.A.B. \& Kent, J. 2000. Biodiversity hotspots for conservation priorities. Nature 403: 853-858.

Nadkarni, N.M. 1992. The conservation of epiphytes and their habitats: Summary of a discussion at the international symposium on the biology and conservation of epiphytes. Selbyana 13: 140-142.

Nadkarni, N.M., Parker, G.G. \& Lowman, M.D. 2011. Forest canopy studies as an emerging field of science. Annals of Forest Science 68: 217-224.

Nieder, J., Engwald, S. \& Barthlott, W. 1999. Patterns of neotropical epiphyte diversity. Selbyana 20: 66-75.

Nieder, J., Prosperi, J. \& Michaloud, G. 2001. Epiphytes and their contribution to canopy diversity. Plant Ecology 153: 51-63.

Parker, G.G. 1995. Structure and microclimate of forest canopies. In: M.D. Lowman \& N.M. Nadkarni (eds.). Forest canopies. Academic Press, San Diego, pp. 73-106.

Poltz, K. \& Zotz, G. 2011. Vascular epiphytes on isolated pasture trees along a rainfall gradient in the lowlands of Panama. Biotropica 43: 165-172.

Prósperi, J., Caballé, G. \& Caraglio, Y. 2001. Lianas and hemiepiphytes: distribution, development, and adaptations. Selbyana 22: 197-212

Schimper, A.F.W. 1888. Die epiphytische Vegetation Amerikas. G. Fischer, Jena.

Schneider, H., Schuettpelz, E., Pryer, K.M., Cranfill, R., Magallón, S. \& Lupia, R. 2004. Ferns diversified in the shadow of angiosperms. Nature 428: 553-557.

Setzer, J. 1966. Atlas Climático e Ecológico do Estado de São Paulo. Comissão Interestadual da Bacia ParanáUruguai, São Paulo.

Smith, J.A.C. 1986. Comparative ecophysiology of crassulacean acid metabolism and 3-carbon pathway bromeliads. 4. Plant water relations. Plant, Cell and Environment 9: 395-410.

Ter Steege, H. \& Cornelissen, J.H.C. 1989. Distribution and ecology of vascular epiphytes in lowland rain forest of Guyana. Biotropica 21: 331-339.

Toppa, R.H. 2004. Estrutura e diversidade florística das diferentes fisionomias de Cerrado e suas correlações com o solo na Estação Ecológica de Jataí, Luiz Antônio, SP. Tese de Doutorado, Universidade Federal de São Carlos, São Carlos.

Tryon, R.M. 1970. Development and evolution of Fern Floras of Oceanic Islands. Biotropica 2: 76-84. 
Waechter, J.L. 1992. O epifitismo vascular na planície costeira do Rio Grande do Sul. Tese de Doutorado, Universidade Federal de São Carlos, São Carlos.

Werneck, M.S. \& Espírito-Santo, M.M. 2002. Species diversity and abundance of vascular epiphytes on Vellozia peresiana in Brazil. Biotropica 34: 51-57.

Whittaker, R.H. 1967. Dominance and diversity in land plant communities. Science 147: 250-260.

Wolf, J.H.D. 1994. Factors controlling the distribution of vascular and nonvascular epiphytes in the northern Andes. Vegetatio 112: 15-28.
Yeaton, R.I. \& Gladstone, D.E. 1982. The pattern of colonization of epiphytes on Calabash trees (Crescentia alata HBK.) in Guanacaste Province, Costa Rica. Biotropica 14: 137-140.

Zar, J.H. 1999. Biostatistical analysis, 4 ed. Prentice Hall, New Jersey.

Zotz, G. \& Schultz, S. 2008. The vascular epiphytes of a lowland forest in Panama-species composition and spatial structure. Plant Ecology 195: 131-141.

Zotz, G., Hietz, P. \& Schmidt, G. 2001. Small plants large plants: the importance of plant size for the physiological ecology of vascular epiphytes. Journal of Experimental Botany 52: 2051-2056. 